\title{
PROPRIEDADES FÍSICAS E MECÂNICAS DA MADEIRA DE ANGELIM-PEDRA SUBMETIDA A TRATAMENTO TÉRMICO
}

\author{
Mayra Daniela Ferreira' \\ Rafael Rodolfo de Melo ${ }^{1,2}$ \\ Leonardo Antônio Moraes Zaque ${ }^{\prime}$ \\ Diego Martins Stangerlin ${ }^{1,3}$
}

\section{Resumo}

O objetivo deste estudo foi avaliar o efeito do tratamento térmico no comportamento das propriedades físicas e mecânicas da madeira de angelim-pedra (Hymenolobium petraeum Ducke). Para isso, foram confeccionadas peças com dimensões de $25,0 \times 9,0 \times 1,7 \mathrm{~cm}$ as quais foram submetidos ao processo de termorretificação, sob diferentes temperaturas tempo de exposição. Utilizaram-se das temperaturas de $180^{\circ} \mathrm{C}$ e $200^{\circ} \mathrm{C}$, com o tempo de exposição variando de 2 as 4 horas, além das amostras testemunhas (sem o tratamento térmico). Após os tratamentos, de cada uma das peças, foram retirados corpos-de-prova para a realização dos ensaios físicos (massa específica, perda de massa, teor de umidade de equilíbrio e estabilidade dimensional) e mecânicos (flexão estática e compressão paralela às fibras). O tratamento térmico proporcionou uma perda de massa da madeira, tendo os tratamentos de maior temperatura apresentado as maiores perdas. Para o teor de umidade de equilíbrio e a estabilidade dimensional o tratamento térmico se mostrou eficaz, melhorando estes parâmetros para todas as peças tratadas termicamente. Quanto as propriedades mecânicas, o tratamento térmico não influenciou a resistência mecânica da madeira.

Palavras-chave: Madeira tropical; Propriedades tecnológicas; Estabilidade dimensional.

\section{EFFECT OF HEAT TREATMENT ON PHYSICAL AND MECHANICAL PROPERTIES OF HYMENOLOBIUM PETRAEUM WOOD}

\begin{abstract}
The work aimed to evaluated the effect of heat treatment on physical and mechanical properties of Hymenolobium petraeum Ducke wood. For this, were manufactured samples with $25.0 \times 9.0 \times 1.7 \mathrm{~cm}$ which were submitted to different heat treatments, with two temperatures $\left(180\right.$ and $200{ }^{\circ} \mathrm{C}$ ) and two exposure times ( 2 and 4 hours), besides the control samples (without the heat treatment). After the treatments, each sample were submitted to the assays with withdrawal of subsamples for the physical (density, mass loss, equilibrium moisture content and dimensional stability) and mechanical properties (static bending and compression parallel to grain). The heat treatment provided a weight loss of wood, with the higher temperature treatments displayed the greatest losses. However, it proved effective to decrease the equilibrium moisture content and the dimensional stability. The mechanical properties were not affected to the heat treatment.
\end{abstract}

Keywords: Tropical wood; Wood properties; Dimensional stability.

\section{INTRODUÇÃO}

A madeira apresenta capacidade de absorção e/ou perda de água para o ambiente, resultando em variações dimensionais que interferem negativamente em sua qualidade. Esse processo é definido como higroscopia e ocorre devido à constituição anatômica e química da madeira. As alterações ocasionadas pelo inchamento e contração da madeira se devem à variação de quantidade de água presente nas paredes celulares [I]

Dentre os principais constituintes da madeira, as hemiceluloses são polímeros hidrofílicos que possuem maior contribuição para o fenômeno de higroscopia, sendo então desejável sua redução e/ou degradação. Para isso, existem

'Programa de Pós-graduação em Ciências Florestais, Universidade Federal de Mato Grosso - UFMT, Cuiabá, MT, Brasil. E-mail: mayradaniela90@gmail.com 2Departamento de Ciências Agrárias e Florestais, Universidade Federal Rural do Semi-Árido - UFERSA, Mossoró, RN, Brasil.

3 Instituto de Ciências Agrárias e Ambientais, Universidade Federal de Mato Grosso - UFMT, Sinop, MT, Brasil. 
diversos tratamentos físicos e químicos que podem utilizados. Dentre estes, o tratamento térmico de madeira, também conhecido como termorretificação, é um dos procedimentos mais utilizados seja para madeira maciça ou em produtos compostos de madeira [2-7].

A madeira termorretificada é obtida por meio do princípio de termodegradação parcial de seus constituintes químicos, na ausência de oxigênio ou forte deficiência de ar. Também pode ser definida como, produto de uma pirólise controlada, a qual é interrompida antes de atingir as reações exotérmicas $[3,6,7]$. Desta forma, além da melhoria na estabilidade da madeira, há a alteração artificial da cor - que pode aumentar a comercialização de espécies de baixa atratividade estética, mas com boas propriedades tecnológicas [8].

Outra vantagem da modificação superficial de peças de madeira pela ação do calor é o seu apelo técnico, econômico e ambiental. Esta técnica pode ser realizada uma única vez e eliminar a aplicação de produtos químicos como vernizes e tintas que demandam aplicações anuais, tornando assim um processo moroso e oneroso [9].

Apesar dos benefícios, o tratamento térmico apresenta desvantagem associada à redução de suas propriedades mecânicas, devido à deterioração de polissacarídeos amorfos e hidrofílicos que compõe a parede celular das células lenhosas, sendo a degradação das hemiceluloses o fator principal da diminuição da resistência [5]. Diante deste contexto, esse estudo tem o objetivo geral de avaliar o efeito da termorretificação nas propriedades tecnológicas da madeira de angelim-pedra (Hymenolobium petraeum).

\section{MATERIAL E MÉTODOS}

\section{I Matéria-prima e Parâmetros Avaliados}

Foram utilizadas peças de madeira com dimensões de $25 \mathrm{~cm} \times 9,0 \mathrm{~cm} \times 1,7 \mathrm{~cm}$, tendo como matéria-prima - Hymenolobium petraeum Ducke (angelim-pedra). Estas foram divididas aleatoriamente em cinco grupos, sendo cada um destes composto por 10 repetições. Posteriormente, estas amostras foram submetidas a diferentes tratamentos térmicos em estufa de circulação forçada de ar, utilizando diferentes tempos e temperaturas de exposição, conforme expresso na Tabela I.

Após os tratamentos, foram retiradas subamostras das peças de madeira para realização dos ensaios físicos e mecânicos. Para avaliação das amostras tratadas e as não tratadas (testemunhas), foram realizados ensaios físicos (massa específica, perda de massa, teor de umidade de equilíbrio e estabilidade dimensional) e mecânicos (resistência à flexão estática e compressão paralela as fibras), adotando as recomendações da Comisión Panamericana de Normas Técnicas - COPANT [10].

Para os ensaios físicos os corpos de prova form dimensionados com $4,9 \mathrm{~cm} \times \mathrm{I}, 7 \mathrm{~cm} \times \mathrm{I}, 7 \mathrm{~cm}$ contendo duas amostras por cada peça tratada, totalizando 20 repetições para cada tratamento. Para os ensaios mecânicos de flexão as amostras foram retiradas nas dimensões de $25 \mathrm{~cm} \times 1,7 \mathrm{~cm} \times 1,7 \mathrm{~cm}$, contendo três amostras por peça tratada, totalizando 30 repetições por tratamento. Já para os ensaios mecânicos de compressão paralela as fibras serão utilizadas peças de $7,0 \mathrm{~cm} \times 1,7 \mathrm{~cm} \times 1,7 \mathrm{~cm}$, contendo duas amostras para cada peça tratada, assim totalizando 20 repetições para cada tratamento.

\subsection{Avaliação das Propriedades Físicas}

A determinação da massa específica aparente da madeira de angelim-pedra foi realizada para todas as amostras submetidas aos ensaios físicos. Para isso, após serem produzidas as amostras foram armazenadas em câmara climatizada, em condições de $20^{\circ} \mathrm{C}$ e $65 \%$ de umidade relativa do ar, até atingirem peso constante (teor de umidade de equilíbrio de 12\%). Após a estabilização das amostras obteve-se a massa e volume das amostras pelo método gravimétrico, com o auxílio de uma balança analítica com precisão de 0,01 g e as dimensões, com a utilização de um paquímetro digital com precisão de $0,01 \mathrm{~mm}$.

A massa especifica foi determinada antes e após as amostras serem submetidas ao tratamento térmico. Adicionalmente, determinou-se a perda de massa das amostras tratadas termicamente. $\mathrm{O}$ teor de umidade de equilíbrio das amostras também foi determinado, antes e após o tratamento térmico.

Para caracterização da estabilidade dimensional, determinaram-se as contrações lineares, a contração volumétrica e o fator anisotrópico para cada uma das amostras. As amostras foram submersas em água, até atingirem peso constante e, em seguidas, foram mensuradas e pesadas. Posteriormente, estas foram encaminhadas à estufa para secagem à $100{ }^{\circ} \mathrm{C} \mathrm{e}$, ainda anidras, determinou-se a sua massa e dimensões secas.

\subsection{Avaliação das Propriedades Mecânicas}

Para determinação das propriedades mecânicas, foi utilizada uma máquina de ensaio tipo universal com capacidade de aplicação de carga de $300 \mathrm{KN}$, com acessórios tais como suportes e equipamento auxiliar para medição automática da carga e deformação das amostras ensaiadas.

Tabela I. Tratamentos térmicos adotados para a madeira de angelim-pedra

\begin{tabular}{ccc}
\hline Tratamento & Tempo (horas) & Temperatura $\left({ }^{\circ} \mathbf{C}\right)$ \\
\hline $0-0$ & - & - \\
$2-180$ & 2 & 180 \\
$2-200$ & 2 & 200 \\
$4-180$ & 4 & 180 \\
$4-200$ & 4 & 200 \\
\hline
\end{tabular}

Tecnol. Metal. Mater. Miner., São Paulo, v. I6, n. I, p. 3-7, jan./mar. 2019 
Os ensaios realizados foram flexão estática e compressão paralela às fibras.

Para determinar o Módulo de Elasticidade e o Módulo de Ruptura das peças, foi realizado o ensaio da flexão estática. $O$ vão utilizado entre os apoios foi de 24 vezes a espessura $(1,7 \mathrm{~cm})$ e a velocidade de aplicação de carga de aproximadamente $2,5 \mathrm{~mm} \mathrm{~min}{ }^{-1}$. $O$ ensaio de flexão estática consistiu em submeter os corpos de prova a uma carga central, até a ruptura. Os corpos de prova foram livremente apoiados, com a carga aplicada por meio de um cutelo central na face tangencial, no centro do vão de $21 \mathrm{~cm}$.

Para avaliar a resistência à compressão paralela, a velocidade utilizada para realização dos ensaios foi de $0,65 \mathrm{~mm} \mathrm{~min}^{-1}$. $O$ ensaio foi realizado na máquina universal de ensaios.

\subsection{Análise dos Resultados}

Os dados obtidos para os parâmetros estudados: massa específica (antes e após o tratamento); contração linear e volumétrica; fator anisotrópico em contração; resistência à flexão estática e à compressão paralela às fibras. Para a análise das diferenças entre os diferentes tratamentos foi realizada a análise de variância ( $5 \%$ de significância), e quando significativo, a comparação entre eles foi feito pelo teste de Scott-Knott, a 5\% de significância.

\section{RESULTADOS E DISCUSSÃO}

\section{I Propriedades Físicas}

Os resultados obtidos de massa específica da madeira de Hymenolobium petraeum variaram entre $0,69 \mathrm{e} 0,71 \mathrm{~g} \mathrm{~cm}^{-3}$ antes da termorretificação, e de 0,67 e $0,70 \mathrm{~g} \mathrm{~cm}^{-3}$ após ○ tratamento térmico (Tabela 2). Não houve diferença significativa na massa específica da madeira de angelim-pedra antes e após a termorretificação ou entre os tratamentos avaliados. Menezes et al. [I I] encontraram resultados semelhantes ao trabalhar com a madeira de $E$. saligna, onde as médias de massa específica encontradas não reduziram significativamente com os tratamentos utilizados.

Observa-se que a madeira termicamente tratada apresentou menor umidade de equilíbrio higroscópico em relação à madeira não tratada (Tabela 2). A diminuição da umidade ocorre devido à menor quantidade de água adsorvida pelas paredes das células como consequência da mudança química, no caso a diminuição de grupos hidroxílicos, e além da diminuição da acessibilidade devido ao aumento da cristalinidade da celulose e aumento das ligações cruzadas na lignina [12].

Podemos perceber a redução da higroscopicidade, aqual pode ser resultado de modificações químicas, em especial a degradação parcial das hemiceluloses $[2,3,4,8]$. No tratamento térmico ocorre uma redução no número de grupos $\mathrm{OH}$ hidrofílicos, que são substituídos por grupos hidrofóbicos O-acetil [13]. Esses grupos são formados pela degradação das hemiceluloses e liberados da madeira na forma de ácido acético [14].

$\mathrm{Na}$ Tabela 3, podem ser observados os valores de contrações lineares e volumétricas para a madeira de angelim pedra, submetidas aos diferentes tratamentos térmicos. Com relação a contração longitudinal e o fator anisotrópico não foram encontradas diferenças significativas entre os tratamentos avaliados. Já para as contrações tangenciais, radiais e volumétricos foram observados diferença significativas, onde os tratamentos que foram submetidos à temperatura de $180^{\circ} \mathrm{C}$ por 2 horas e 4 horas, apresentaram os menores valores, sendo estes considerados os mais estáveis.

Os resultados obtidos para as variações dimensionais (contrações) da madeira indicaram uma melhoria significativa

Tabela 2. Valores médios de massa especifica, perda de massa e teor de umidade de equilíbrio antes e após do tratamento térmico da madeira de angelim-pedra

\begin{tabular}{|c|c|c|c|}
\hline Tempo-Temperatura & \multicolumn{2}{|c|}{ Massa Específica $\left(\mathrm{g} \mathrm{cm}^{-3}\right)^{\mathrm{Ns}}$} & \multirow{2}{*}{ Perda de Massa (g) } \\
\hline (horas- $\left.{ }^{\circ} \mathbf{C}\right)$ & Antes & Após & \\
\hline $0-0$ & 0,712 & 0,716 & $0,00 \mathrm{~d}$ \\
\hline $2-180$ & 0,694 & 0,680 & $\mathrm{I}, 93 \mathrm{~b}$ \\
\hline $2-200$ & 0,708 & 0,700 & $3,47 c$ \\
\hline $4-180$ & 0,704 & 0,710 & $2,04 \mathrm{~b}$ \\
\hline $4-200$ & 0,690 & 0,679 & $5,55 \mathrm{a}$ \\
\hline Tempo-Temperatura & \multicolumn{3}{|c|}{ Teor de Umidade de Equilíbrio (\%) } \\
\hline (horas- $\left.{ }^{\circ} \mathbf{C}\right)$ & \multicolumn{2}{|c|}{ Antes } & Após \\
\hline $0-0$ & \multicolumn{2}{|c|}{$\mathrm{II}, 49 \mathrm{aA}$} & $\mathrm{II}, 49 \mathrm{aA}$ \\
\hline $2-180$ & \multicolumn{2}{|c|}{$11,28 \mathrm{aA}$} & $8,70 \mathrm{bB}$ \\
\hline $2-200$ & \multicolumn{2}{|c|}{$\mathrm{I} I, 45 \mathrm{aA}$} & $7,79 \mathrm{bB}$ \\
\hline $4-180$ & \multicolumn{2}{|c|}{$\mathrm{I} I, 40 \mathrm{aA}$} & $7,68 \mathrm{bB}$ \\
\hline $4-200$ & \multicolumn{2}{|c|}{$11,36 \mathrm{aA}$} & $7,54 \mathrm{bB}$ \\
\hline
\end{tabular}

${ }^{\text {Ns }}$ não significativo; Médias seguidas por uma mesma letra minúscula na vertical, ou maiúscula no horizontal, não diferem estatisticamente (Scott-Knott, $\left.\alpha=0,05\right)$. 
Tabela 3. Valores médios de contrações lineares, contrações volumétricas e fator anisotrópico para os diferentes tratamentos avaliados

\begin{tabular}{|c|c|c|c|c|c|}
\hline \multirow{2}{*}{$\begin{array}{c}\text { Tempo-Temperatura } \\
\left.\text { (horas- }{ }^{\circ} \mathbf{C}\right)\end{array}$} & \multicolumn{4}{|c|}{ Contração (\%) } & \multirow{2}{*}{ fa } \\
\hline & $\beta \mathbf{I}$ & $\beta r$ & $\beta \mathbf{t}$ & $\beta \mathbf{v}$ & \\
\hline $0-0$ & $0,48 a$ & $4,84 \mathrm{a}$ & $6,47 \mathrm{a}$ & $11,33 \mathrm{a}$ & $1,38 \mathrm{a}$ \\
\hline $2-180$ & $0,43 a$ & $2,88 d$ & $4,7 \mid \mathrm{c}$ & $7,45 \mathrm{~d}$ & $1,35 \mathrm{a}$ \\
\hline $2-200$ & $0,4 \mathrm{I} a$ & $3,69 c$ & $5,07 \mathrm{~b}$ & $8,77 \mathrm{c}$ & $1,39 a$ \\
\hline $4-180$ & $0,52 \mathrm{a}$ & $3,26 c$ & $4,89 c$ & $8,10 \mathrm{c}$ & $I, 4 I a$ \\
\hline $4-200$ & $0,36 \mathrm{a}$ & $4,31 \mathrm{~b}$ & $6,12 b$ & $10,37 \mathrm{~b}$ & $\mathrm{I}, 50 \mathrm{a}$ \\
\hline
\end{tabular}

$\beta$ i: contração longitudinal; $\beta \mathrm{r}$ : contração radial; $\beta \mathrm{t}$ : contração tangencial; $\beta \mathrm{v}$ : contração volumétrica; fa: fator anisotrópico; Médias seguidas por uma mesma letra minúscula na vertical, não diferem estatisticamente (Scott-Knott, $\alpha=0,05)$.

Tabela 4. Valores médios de módulo de elasticidade (MOE) e módulo de ruptura (MOR) aos ensaios de flexão estática e compressão paralela as fibras, das amostras submetida aos diferentes tratamentos térmicos

\begin{tabular}{cccccc}
\hline $\begin{array}{c}\text { Tempo-Temperatura } \\
\left.\text { (horas- }{ }^{\circ} \mathbf{C}\right)\end{array}$ & \multicolumn{2}{c}{ Flexão Estática (MPa) } & & & \multicolumn{2}{c}{ Compressão Paralela (MPa) $^{\text {NS }}$} \\
\cline { 2 - 3 } \cline { 5 - 6 } & MOE & MOR & 67,18 & & MOE Ns \\
$2-180$ & 11.107 & 70,76 & 14.940 & 55,66 \\
$2-200$ & 11.767 & 71,38 & 14.900 & 56,83 \\
$4-180$ & 12.234 & 71,94 & 15.600 & 57,97 \\
$4-200$ & 11.062 & 67,09 & 14.100 & 57,38 \\
\hline
\end{tabular}

na estabilidade dimensional. Dessa forma, comparando-se as variações dimensionais nas diferentes direções estruturais, observa-se que os valores de contração na direção tangencial são maiores que na direção radial, os quais, por sua vez, são maiores que na direção longitudinal. Isso ocorre, em decorrência da anisotropia da madeira. As variações dimensionais no sentido transversal (tangencial e radial) são maiores que no sentido longitudinal, já que a maioria das células está orientada longitudinalmente [I]. No sentido radial, as variações dimensionais são menores que no sentido tangencial devido à orientação transversal dos raios e da presença de pontuações areoladas predominante nas paredes radiais das fibras/traqueoides, causando um desvio das microfibrilas de celulose na camada S2.

\subsection{Propriedades Mecânicas}

Os resultados da análise estatística para o módulo de elasticidade e o módulo de ruptura no ensaio de flexão estática não apresentaram diferenças significativas (Tabela 4).

A não influência do tratamento térmico na resistência da madeira pode ser atribuída a utilização de uma temperatura mais amena, insuficiente para degradar expressivamente os componentes estruturais a madeira e também pela utilização de atmosfera livre de oxigênio que pode ter ocasionado um rearranjo da estrutura química da madeira tornando-a um pouco mais resistente [15-I7].

Os valores médios do módulo elasticidade e do módulo de ruptura em compressão paralela às fibras também não apresentaram diferença significativa no teste de compressão paralela as fibras (Tabela 4). Resultados semelhantes foram observados por Xavier [18] avaliando dois níveis de temperatura $\left(180^{\circ} \mathrm{C}, 200^{\circ} \mathrm{C}\right)$ e dois níveis de tempo de exposição (I e 2 horas). Segundo o autor, estes tratamentos não exerceram influência significativa na resistência e rigidez a compressão das madeiras de Pinus sp. e do híbrido Eucalyptus urograndis.

Estes resultados corroboram com Vernois [19], que afirma que para as espécies tratadas em temperaturas até $210^{\circ} \mathrm{C}$, as propriedades mecânicas permanecem com valores próximos aos originais. Nesse mesmo sentido, Yildiz et al. [20] examinando as mudanças na estrutura química, celulose, hemiceluloses e lignina, da madeira tratada sob diferentes temperaturas e duração do tratamento térmico, concluiram que o conteúdo de lignina, ao contrário da celulose e hemiceluloses, exibiu um aumento com os tratamentos mais agressivos.

\section{CONCLUSÃO}

O tratamento térmico acarretou uma pequena perda de massa de acordo com o aumento da temperatura e tempo de exposição. Em contrapartida, o processo resultou em melhorias na estabilidade dimensional da madeira de angelim-pedra, além de proporcionar uma redução no teor de umidade de equilíbrio das amostras. Para as propriedades mecânicas não foram verificadas alterações. Os melhores resultados foram observados na aplicação da temperatura $180^{\circ} \mathrm{C}$ com o tempo de exposição de 2 horas. 


\section{REFERÊNCIAS}

I Jones P. Wood and water. USA: Wiley Blackwell; $201 \mathrm{I}$.

2 Kamdem DP, Pizzi A, Jermannaud A. Durability of heat-treated wood. Holz als Roh- und Werkstoff. 2002;60:I-6.

3 Brito JO, Garcia JN, Bortoletto G Jr, Pessoa AMC, Silva PHM. Densidade básica e retratibilidade da madeira de Eucalyptus grandis, submetida a diferentes temperaturas de termorretificação. Cerne. 2006; I2: I82- I88.

4 Esteves B, Domingos I, Pereira $\mathrm{H}$. Improvement of technological quality of eucalypt wood by heat treatment in air at $170-200^{\circ} \mathrm{C}$. Forest Products Journal. 2007;57:47-52.

5 Garcia RA, Carvalho AM, Latorraca JVF, Matos JLM, Santos WA, Silva RFM. Nondestructive evaluation of heattreated Eucalyptus grandis Hill ex Maiden wood using stress wave method. Wood Science and Technology. 20I2;46:4I-52.

6 Lopes JO, Garci RA, Nascimento AM, Latorraca JVF. Uniformização da cor da madeira jovem de teca pela termorretificação. Revista Árvore. 2014;38:56I-568.

7 Wandscheer RB, Bressan J, Melo RR, Lima DC, Pedrosa TD, Ferreira MD. Thermal treatment effect on physical and mechanical properties of MDF panels. Nativa. 2016;4:71-76.

8 Borges LM, Quirino WF. Higroscopicidade da madeira de Pinus caribaea var. hondurensis tratado termicamente. Biomassa \& Energia. 2004; I:173-182.

9 Gouveia FN. Aplicação de tratamentos térmicos para estabilização colorimétrica de madeiras tropicais [Doutor em Ciência Florestal]. Brasília: Universidade de Brasília; 2008.

10 Comisión Panamericana de Normas Técnicas - COPANT. Caracterización tecnológica de las maderas: normas 458; 459; 460; 46I; 462; 463; 555. La Paz: COPANT, 1972.

II Menezes WM, Santini EJ, Souza JT, Gatto DA, Haselein CR. Modificação térmica nas propriedades físicas da madeira. Ciência Rural. 2014;44:1019-1024.

12 Boonstra MJ, Tjeerdsma BF. Chemical analysis of heat treated softwoods. Holz als Roh- und Werkstoff. 2006;64:204-2II.

13 Poncsák S, Kocaefe D, Bouazara M, Pichette A. Effect of high temperature treatment on the mechanical properties of birch (Betula papyrifera). Wood Science and Technology. 2006;40:647-663.

14 Sundqvist B, Morén T. The influence of wood polymers and extractives on wood color induced by hydrothermal treatment. Holz als Roh- und Werkstoff. 2002;60:375-376.

I5 Boonstra MJ, Acker JV, Tjeerdsma BF, Kegel EV. Strength properties of thermally modified softwoods and its relation to polymeric structural wood constituents. Annals of Forest Science. 2007;64:679-690.

16 Gunduz G, Aydemir D. Some physical properties of heat-treated hornbeam (Carpinus betulusL.) wood. Drying Technology. 2009;27:7I4-720.

17 Soratto, D. N. Efeito do tratamento térmico nas propriedades da madeira de Eucalyptus sp. [dissertação]. Viçosa: Universidade Federal de Viçosa; 2012.

18 Xavier GS. Influência de tratamentos térmicos em duas propriedades mecânicas das madeiras de Pinus sp. e Eucalyptus urograndis [monografia]. Brasília: Universidade Federal de Brasília; 2013.

19 Vernois M. Heat treatment of wood in France - stat of the art. Paris: Centre Technique du Bois et de l'Ameublement; 2000.

20 Yildiz S, Gezer ED, Yildiz UC. Mechanical and chemical behavior of spruce wood modified by heat. Building and Environment. 2006;41:1762-1766.

Recebido em: 17 Jan. 2017

Aceito em: 14 Abr. 2018 